\title{
EFFECT OF IRRIGATION MANAGEMENT AND SEEDLING AGE ON THE PRODUCTIVITY AND QUALITY OF TWO RICE VARIETIES USING SYSTEM OF RICE INTENSIFICATION (SRI).
}

\author{
El-Khoby. M. W. ${ }^{\star}$ and Alaa.M.E. A. Shahein ${ }^{\star \star}$ \\ ${ }^{*}$ Rice Research Section. Field Crops Res Institute, ARC, Egypt \\ ${ }^{*}$ Seed Technology Res. Dept, Field Crops Res. Institute, ARC., Egypt
}

\begin{abstract}
A two -year field experiment was conducted at the Experimental Farm of Agricultural Research Station and lab. of seed technology Dept. Sakha, Kafr El-Sheikh, Egypt, during 2013 and 2014 seasons. A split split plot design with three replications was conducted to study the effect of irrigation system, seedling age and variety on rice yield and its components, grain quality and viability. The two irrigation treatments i.e. continues flooding and continues saturation were allocated to the main plots, three ages of seedlings i.e. 15, 20 and 25 days old were assigned to the sub-plots and two rice varieties i.e., Sakha105 as inbred and Egyptian hybrid1 rice were distributed in sub-sub plots. The obtained results could be summarized as follows:

The grain yield, straw yield, hectolitre weight, protein content, hulling \% and milling \% responded positively to flooding treatment. Transplanting of younger seedlings provided more effective tillers $\mathrm{m}^{-1}$, filled grains panicle ${ }^{-1}$, thousand grain weight, hectolitre weight, straw yield, germination, protein content and amylose content, hulling $\%$, milling $\%$ and grain yield than those of the older one. Egyptian Hybrid 1 rice produced the highest values of number of productive tillers hill, number of primary branches/panicle, grain yield, straw yield, radical length, E.C and 1000-grain weight. The interactions among irrigation, age of seedling and rice variety had a significant effect on number of productive tillers $/ \mathrm{m}^{2}$, number of primary branches/panicle, filled grains, panicle weight, straw yield, germination \%, E.C and 1000-grain weight.

The present study indicated that sowing of Egyptian Hybrid 1 rice variety with 15 days seedlings old under flooding treatment can improve yield and its components as well as grain quality of economic values.
\end{abstract}

Key words: Rice, irrigation treatments, seedling age, cultivars, viability and quality.

\section{INTRODUCTION}

Rice is the most water-consuming crop grown in Egypt, occupying around 0.6 million hectares of land under continuous flooding during the growing season. Water availability is becoming progressively more limited as an increasing population creates competing demands for this precious resource. The challenge for agricultural researchers is to find ways to reduce water used in rice production while continuing to increase yields. There is also a need to understand the impacts of 
growing rice with less water on labor requirements, weed control, fertilizer management, cultural practices, and rice grain quality. An additional issue for Egypt is that farmers sometimes use irrigation water containing elevated levels of heavy metals, which could degrade the quality of rice and other crops.

Rice production in Egypt, occupies 22\% of cultivated area in the summer season, and consumes $13 \%$ of total water. Egypt presently has the highest average national rice yield in the world; however, the country's rice output must increase by $20 \%$ over the next decade just to maintain current levels of consumption. This will be difficult because the yield level is already high, and because of increasing competition for water with growing water shortages that affect all sectors. SRI methods for growing more rice with less water thus warrant evaluation under Egyptian conditions.

Rice cultivars performed differently when transplanted in field at varying seedling ages depending upon their genetic makeup and adoptability to certain environmental conditions. Seedling age plays an important role in yield contributing parameters like number of productive tillers, panicle length, filled grains panicle ${ }^{-1}$ and 1000-kernel weight leading to higher paddy yield in different rice cultivars and hybrids.

The System of Rice Intensification (SRI) is one of the best alternatives to minimize the water consumption for the cultivation of rice and at the same time increasing the productivity. System of Rice Intensification (SRI), a recently method in rice to increase its productivity was developed in Madagascar in the 1980,s, due to its less consumption of water, nearly $50 \%$ as compared to the other conventional methods. The cultivation under SRI is rapidly increasing. $\mathrm{SRI}$ is a set of ideas and insights that emphasize the use of younger seedlings (less than 15 days) planted singly and intermittent irrigation, together with the adoption of at wider spacing, organic fertilization, and active soil aeration to the extent possible (Uphoff, 2007; Stoop et al., 2002). With this backdrop, an experiment was taken up to identify the correct irrigation system, age of the seedlings and rice cultivars for realizing good quality seed under SRI system of cultivation.

Transplanting rice seedlings from a nursery into the paddy field when the plants are relatively mature, 3 to 4 weeks old, as is common practice around the world, with SRI seedlings are transplanted before they are 15 days old, even 8 or 10 days old. This preserves the plants' potential for massive tillering if the other practices are followed. Transplanting seedlings in clumps of 3 or 4 , as is almost universally done, with SRI seedlings are transplanted singly, so that there is no competition among plant roots to inhibit growth. Keeping paddy fields continually flooded, with SRI soils are kept wellaerated during the vegetative growth phase. the increase in the yield with SRI was 
attributed to the increase in number of ear bearing tillers /hill, total number of spikelet /panicle and total length, Rafaralahy (2002).

\section{MATERIALS AND METHODS}

A two -year field experiment was conducted at the Experimental Farm of Sakha Agricultural Station and lab. of seed technology Dept. Sakha, Kafr El-Sheikh, Egypt, during 2013 and 2014 rice growing seasons. The experiments were conducted to investigate the response of two rice varieties, i.e., Sakha105 and Egyptian hybrid 1 to irrigation treatment and seedling age on yield and its components, grain quality and viability. The experimental design was split split plot with three replications. The main plot was randomly assigned to two irrigations system i.e. continues flooding and continues saturation. Three sub-plots were assigned to seedling age i.e. 15, 20 and 25 days old seedling with two sub-sub plots for rice varieties i.e. Sakha105 and Egyptian hybrid 1 rice. Seeds of rice Sakha105 and Egyptian Hybrid 1 rice cultivars at the rate of 40 and $10 \mathrm{~kg} / \mathrm{fed}$, respectively, were planted dry seed on dry soil and then irrigated on May $10^{\text {th }}$ in both seasons. Seedlings from the different aged nurseries were removed according to the assigned treatments and 3-5 seedlings per hill were transplanted in $20 \times 20 \mathrm{~cm}$ spacing into $15 \mathrm{~m}^{2}$ plot size. The nitrogen fertilizer in the form of urea from $(46 \% \mathrm{~N})$ was applied as assigned levels in two splits i.e. 2/3 as basal application and $1 / 3$ as topdressing at panicle initiation stage. The recommended doses of the other fertilizers i.e. phosphorus as single super phosphate $\left(15.5 \% \mathrm{P}_{2} \mathrm{O}_{5}\right)$ was added at the rate of $100 \mathrm{~kg} / \mathrm{fed}$. and $\mathrm{zn}$ sulphate $(22 \% \mathrm{Zn})$ at the rate of $20 \mathrm{~kg} / \mathrm{fed}$. was added after pudding before planting.

\section{Data collection}

Important parameters like number of tillers $/ \mathrm{m}^{2}$, number of primary branches/panicle, filled grains/panicle, panicle weight (g), 1000grain weight, grain yield (t/ha) and straw yield (t/ha) were recorded from ten randomly selected plants from each replication to assess treatment effects on crop growth and performance. The grains obtained from all the treatments were tested for laboratory germination which was conducted according to international rules (ISTA, 1993). Electrical conductivity (EC) was measured according to (AOSA, 1986). Hectolitre weight $(\mathrm{g} h \mathrm{~h}-1)$ : it was determined for dockage-free grain sample using seedburo hectolitre mass device and electronic balance. The protein content was determined according to the procedures outlined in (A.O.A.C, 1990). The amylose content was determined according to the iodimetric technique (Blue Value), described by Gilbert \& Spragg (1964). Hulling percentage: About $150 \mathrm{~g}$ cleaned rough rice samples at moisture content $12.14 \%$ were estimated using experimental huller machine (Stake) in Rice Research and Training Center, grain quality lab 
Hulling $\%=$ Weight of brown rice/ weight of rough rice $\times 100$. Milling percentage: Brown rice was consequently milled using McGILL Millerno2. The sample was milled for $60 \mathrm{sec}$. the milled rice sample was then collected and the weight was taken and percentage of total milled rice was computed.

Milled rice $\%=$ Weight of milled rice/weight of rough rice $\times 100$

Data collected in the two seasons were statistically analyzed according to the technique of analysis of variance (ANOVA) for three factors in split split plot. For comparison between means, LSD at $0.05 \%$ of probability was used. All data were calculated according to Dunacn (1955). All statistical analyses were performed using analyses of variance technique by "MSTAT-C" computer software package (1990).

\section{RESULTS AND DISSCUSSION}

Pooled data in both seasons as shown in Tables 1, 2, 3,4,5 and 6 revealed significant differences in productivity and grain quality of the two rice varieties due to the irrigation treatment, age of seedlings, and their interactions.

\section{Effect of irrigation treatments:}

Irrigation treatment significantly affected number of productive tillers $/ \mathrm{m}^{2}$ and number of branches/panicle only in the first season as shown in Table (1). Saturation treatment gave the highest number of productive tillers $/ \mathrm{m}^{2}$ and number of branches/panicle in the first season, but the differences in the second season did not reach the $5 \%$ level of significant with flooding treatment. Saturation treatment trended to decrease number of filled grains/panicle, but the differences with flooding treatment did not reach the $5 \%$ level of significant in both seasons. Saturation as a system of irrigation recorded the highest panicle weight $(3.49 \mathrm{gm})$ while, flooding irrigation showed posteriority regarding panicle weight in the second seasons of study in Table (2). Flooding system indicates considerable superiority in grain yield in the first season and straw yield in both seasons compared with saturation system. The present finding is in a good agreement with those reported by Singh et al (1998), Ginigaddara and Ranamukhaarachchi (2011), El-Feky (2012), Ali et al (2013) and Shantappa et al (2014)

Data in Tables ( 3 and 4) demonstrate there were no significant differences between irrigation systems in both studied seasons at germination \%. The flooding irrigation gave the lowest conductivity in the first season. While, flooding gave the highest hectoliter weight $(75.09$ and $74.63 \mathrm{~g})$ in both seasons. Flooding system had the highest values for protein content (7.99 and 7.94\%), hulling \% (85.40 and $84.70 \%)$ and milling\% (63.20 and $63.27 \%)$ in both seasons, 
respectively. The present findings are in complete agreement with those reported by El-Kady and Abdallah (2004).

\section{Effect of seedlings age:}

Data presented in Tables (1 and 2) show clearly that seedling age caused a highly significant effect on number of productive tiller $/ \mathrm{m}^{2}$, number of filled grains/panicle, grain yield and straw yield, but failed to excrete any significant effect on number of branches/panicle and panicle weight in the two seasons of study. 15 days age of seedling resulted in the highest number of productive tillers $/ \mathrm{m}^{2}$, number of filled grains/panicle, grain yield (11.73 and $12.76 \mathrm{t} / \mathrm{ha}$ ) and straw yield (16.06 and $15.39 \mathrm{t} / \mathrm{ha}$ ) without significant differences with 20 days age of seedling in most cases in both seasons. Overall younger seedlings produced higher number of tillers than older seedling, which might be due to less root damage and minimal transplanting shock, as younger seedlings can more easily establish themselves after transplanting in the main field and conscuntly produced more grains/panicle. These results are in accord with those reported by El-Rewainy et al (2007), Krishna et al (2008), Wong et al (2008), Naeem et al (2011), Salem et al (2011), El-Fky (2012), El-Hefnawy (2012), Leila et al (2013), Aggarwal et al (2014), Rajendran and Gameasa (2014), Duraga et al (2015) and Akhilesh et al (2016).

Table (1): Number of productive tillers $/ \mathrm{m}^{2}$, number of branches/ panicle and number of filled grains/ panicle of the two rice varieties as affected by irrigation treatments and seedling age and varieties in 2013 and 2014 seasons.

\begin{tabular}{|c|c|c|c|c|c|c|}
\hline \multirow[t]{2}{*}{$\begin{array}{l}\text { Characters } \\
\text { Treatment }\end{array}$} & \multicolumn{2}{|c|}{$\begin{array}{c}\text { No of productive } \\
\text { tillers } / \mathrm{m}^{2}\end{array}$} & \multicolumn{2}{|c|}{$\begin{array}{l}\text { No. of primary branches/ } \\
\text { panicle }\end{array}$} & \multicolumn{2}{|c|}{ No. of filled grains/ panicle } \\
\hline & 2013 & 2014 & 2013 & 2014 & 2013 & 2014 \\
\hline $\begin{array}{l}\text { Irrigation (I): } \\
\text { Flooding } \\
\text { Saturation } \\
\text { F. test }\end{array}$ & 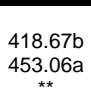 & $\begin{array}{l}408.56 \\
425.67 \\
\text { NS }\end{array}$ & $\begin{array}{l}8.97 \mathrm{~b} \\
9.20 \mathrm{a} \\
*\end{array}$ & $\begin{array}{l}9.41 \\
9.53 \\
\text { NS }\end{array}$ & $\begin{array}{l}140.33 \\
134.52 \\
\text { NS }\end{array}$ & $\begin{array}{l}141.57 \\
137.62 \\
\text { NS }\end{array}$ \\
\hline $\begin{array}{c}\text { Seedling age }(\mathrm{S}): \\
15 \text { days } \\
20 \text { days } \\
25 \text { days } \\
\text { F. test }\end{array}$ & $\begin{array}{l}447.17 \mathrm{a} \\
422.50 \mathrm{~b} \\
397.92 \mathrm{~b} \\
\star \star\end{array}$ & $\begin{array}{c}446.33 a \\
439.00 a \\
401.00 b \\
\star \star\end{array}$ & $\begin{array}{l}9.33 \\
9.27 \\
8.66 \\
\text { NS }\end{array}$ & $\begin{array}{l}9.46 \\
9.55 \\
9.40 \\
\text { NS }\end{array}$ & $\begin{array}{l}144.18 \mathrm{a} \\
140.18 \mathrm{a} \\
127.83 \mathrm{~b} \\
\star *\end{array}$ & $\begin{array}{c}145.74 \mathrm{a} \\
140.06 \mathrm{ab} \\
133.00 \mathrm{~b} \\
\star \star \star\end{array}$ \\
\hline $\begin{array}{c}\text { Rice variety }(\mathrm{V}): \\
\text { Sakha105 } \\
\text { Hybrid } 1 \\
\text { F. test }\end{array}$ & $\begin{array}{l}400.28 \mathrm{~b} \\
\underset{\star \star \star}{423.44 \mathrm{a}}\end{array}$ & $\begin{array}{c}404.44 b \\
429.78 a \\
\star *\end{array}$ & $\begin{array}{l}9.05 \\
9.12 \\
\text { NS }\end{array}$ & $\begin{array}{l}9.28 \mathrm{~b} \\
9.66 \mathrm{a} \\
\star \star\end{array}$ & $\begin{array}{l}137.29 \\
137.57 \\
\text { NS }\end{array}$ & $\begin{array}{l}137.37 \\
141.83 \\
\text { NS }\end{array}$ \\
\hline $\begin{array}{c}A \times B \\
A \times C \\
B \times C \\
A \times B \times C\end{array}$ & $\begin{array}{l}* * \\
* * \\
* * \\
\star *\end{array}$ & $\underset{* *}{* *}$ & $\begin{array}{c}\text { NS } \\
* \\
\text { NS } \\
* *\end{array}$ & $\begin{array}{l}\text { NS } \\
\star \star \\
\star \star \\
\text { NS }\end{array}$ & $\begin{array}{l}\text { NS } \\
\star * \\
\text { NS } \\
* *\end{array}$ & $\begin{array}{l}* * \\
\star * \\
\star * \\
\star *\end{array}$ \\
\hline
\end{tabular}

${ }^{*},{ }^{* *}$ and NS indicated $\mathrm{P}<0.05 \%, \mathrm{P}<0.01 \%$ and not significant, respectively. Means followed by the same letter are not significantly different at the $5 \%$ level, using Duncan's multiple range test. 
Table (2): Panicle weight (g), grain yield (t/ha) and straw yield $(t / h a)$ of the two rice varieties as affected by irrigation treatment and seedling age in 2013 and 2014 seasons.

\begin{tabular}{|c|c|c|c|c|c|c|}
\hline \multirow{2}{*}{$\begin{array}{l}\text { Characters } \\
\text { Treatment }\end{array}$} & \multicolumn{2}{|c|}{ Panicle weight (g) } & \multicolumn{2}{|c|}{ Grain yield (t/ha) } & \multicolumn{2}{|c|}{ Straw yield (t/ha) } \\
\hline & 2013 & 2014 & 2013 & 2014 & 2013 & 2014 \\
\hline $\begin{array}{l}\text { Irrigation (I): } \\
\text { Flooding } \\
\text { Saturation } \\
\text { F. test }\end{array}$ & $\begin{array}{l}3.39 \\
3.50 \\
\text { NS }\end{array}$ & $\begin{array}{l}3.33 b \\
3.49 a \\
\star *\end{array}$ & $\begin{array}{l}10.97 \mathrm{a} \\
10.43 \mathrm{~b} \\
\star *\end{array}$ & $\begin{array}{c}11.54 \\
11.14 \\
\text { NS }\end{array}$ & $\begin{array}{l}13.27 \mathrm{a} \\
12.71 \mathrm{~b} \\
\star *\end{array}$ & $\begin{array}{l}14.67 \mathrm{a} \\
13.69 \mathrm{~b} \\
\star \star\end{array}$ \\
\hline $\begin{array}{c}\text { Seedling age }(\mathrm{S}): \\
15 \text { days } \\
20 \text { days } \\
25 \text { days } \\
\text { F. test }\end{array}$ & $\begin{array}{c}3.47 \\
3.47 \\
3.39 \\
\text { NS }\end{array}$ & $\begin{array}{c}3.63 \\
3.38 \\
3.21 \\
\text { NS }\end{array}$ & 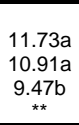 & $\begin{array}{c}12.76 \mathrm{a} \\
11.33 \mathrm{~b} \\
9.93 \mathrm{c} \\
\star \star\end{array}$ & $\begin{array}{l}16.06 \mathrm{a} \\
15.73 \mathrm{a} \\
11.70 \mathrm{~b} \\
\star * *\end{array}$ & $\begin{array}{l}15.39 \mathrm{a} \\
15.28 \mathrm{a} \\
11.88 \mathrm{~b} \\
\star \star\end{array}$ \\
\hline $\begin{array}{c}\text { Rice varieties (V): } \\
\text { Sakha105 } \\
\text { Hybrid } 1 \\
\text { F. test }\end{array}$ & $\begin{array}{l}3.42 \\
3.47 \\
\text { NS }\end{array}$ & $\begin{array}{l}3.37 \\
3.46 \\
\text { NS }\end{array}$ & $\begin{array}{c}10.45 \\
12.95 \\
\text { NS }\end{array}$ & $\begin{array}{l}11.22 \mathrm{~b} \\
12.46 \mathrm{a} \\
\star *\end{array}$ & $\begin{array}{l}13.81 \mathrm{~b} \\
15.18 \mathrm{a} \\
\star \star *\end{array}$ & $\begin{array}{c}13.04 b \\
15.33 a \\
* *\end{array}$ \\
\hline $\begin{array}{c}A \times B \\
A \times C \\
B \times C \\
A \times B \times C\end{array}$ & $\begin{array}{l}\text { NS } \\
\text { NS } \\
\text { NS } \\
\text { NS }\end{array}$ & $\begin{array}{l}\text { NS } \\
\text { NS } \\
* *\end{array}$ & $\begin{array}{l}* \\
* \\
\text { NS } \\
\text { NS }\end{array}$ & $\begin{array}{l}\text { NS } \\
\text { NS } \\
\text { NS } \\
\text { NS }\end{array}$ & $\begin{array}{l}* * \\
* * \\
* * \\
* *\end{array}$ & $\begin{array}{l}* \star \\
* * \\
* * \\
* \star\end{array}$ \\
\hline
\end{tabular}

${ }^{*},{ }^{* *}$ and NS indicated $\mathrm{P}<0.05 \%, \mathrm{P}<0.01 \%$ and not significant, respectively. Means followed by the same letter are not significantly different at the $5 \%$ level, using Duncan's multiple range test.

Data in Tables (3 and 4) show that there were significant difference among seedling age regarding germination \%, 1000-grain weight, hectolitre weight, protein $\%$, amylose $\%$, hulling $\%$ and milling $\%$. Fifteen days old seedling gave the highest values than other treatments. However EC values were decreased with the same treatments. These results are in line with those reported by Singh et al (2004), Krishna et al (2008), Wong et al (2008), El-Hefnawy (2012), Ali et al (2013), Naidu et al (2013) and Pramanik and Bera (2013).

\section{Effect of varieties:}

Data illustrated in Tables (1 and 2) confirmed that there were highly significant differences between the two tested rice varieties on number of productive tillers $/ \mathrm{m}^{2}$ and straw yield in both seasons

Table (3): Laboratory germination, electrical conductivity ( $\mu$ hos/g), 1000-grain weight (g) and hectolitre weight $\left(\mathrm{hl}^{-1}\right)$ of the two rice varieties as affected by irrigation treatment and seedling age in 2013 and 2014 seasons.

\begin{tabular}{|c|c|c|c|c|c|c|c|c|}
\hline \multirow[t]{2}{*}{$\begin{array}{l}\text { Characters } \\
\text { Treatment }\end{array}$} & \multicolumn{2}{|c|}{ Lab. Germination \% } & \multicolumn{2}{|c|}{$\mathrm{EC}(\mu \mathrm{hos} / \mathrm{g})$} & \multicolumn{2}{|c|}{ 1000-Grain weight (g) } & \multicolumn{2}{|c|}{ Hectolitre weight (hl-1) } \\
\hline & 2013 & 2014 & 2013 & 2014 & 2013 & 2014 & 2013 & 2014 \\
\hline $\begin{array}{l}\text { Irrigation (I): } \\
\text { Flooding } \\
\text { Saturation } \\
\text { F. test }\end{array}$ & $\begin{array}{c}83.56 \\
77.11 \\
\text { NS }\end{array}$ & $\begin{array}{c}85.11 \\
77.56 \\
\text { NS }\end{array}$ & $\begin{array}{c}7.18 \mathrm{~b} \\
7.76 \mathrm{a} \\
\star \star\end{array}$ & $\begin{array}{c}6.76 \\
7.04 \\
\text { NS }\end{array}$ & $\begin{array}{c}25.45 \\
25.30 \\
\text { NS }\end{array}$ & $\begin{array}{c}25.45 \\
25.27 \\
\text { NS }\end{array}$ & $\underset{\star \star \star}{75.09 a}$ & $\begin{array}{c}74.63 \mathrm{a} \\
73.41 \mathrm{~b} \\
\star \star \star\end{array}$ \\
\hline $\begin{array}{l}\text { Seedling age }(\mathbf{S}): \\
15 \text { days } \\
20 \text { days } \\
25 \text { days } \\
\text { F. test }\end{array}$ & $\begin{array}{l}86.33 a \\
84.00 a \\
70.67 b \\
\star \star\end{array}$ & $\begin{array}{c}86.67 \mathrm{a} \\
81.67 \mathrm{ab} \\
\underset{\star}{76.00 \mathrm{~b}} \\
\star \star\end{array}$ & $\begin{array}{l}7.19 \mathrm{~b} \\
7.38 \mathrm{~b} \\
7.85 \mathrm{a} \\
\star \star \\
\end{array}$ & $\begin{array}{l}6.68 \mathrm{~b} \\
6.83 \mathrm{~b} \\
7.19 \mathrm{a} \\
\star \star \\
\end{array}$ & $\begin{array}{l}26.24 a \\
24.97 b \\
24.92 b \\
\star \star \star\end{array}$ & $\begin{array}{c}25.71 \mathrm{a} \\
25.46 \mathrm{ab} \\
\underset{\star \star \star}{24.91 \mathrm{~b}}\end{array}$ & $\begin{array}{c}75.14 a \\
74.48 b \\
73.83 c \\
\star \star \star\end{array}$ & $\begin{array}{c}74.68 \mathrm{a} \\
74.02 \mathrm{~b} \\
73.37 \mathrm{c} \\
{ }_{\star \star} \\
\end{array}$ \\
\hline $\begin{array}{l}\text { Rice varieties (V): } \\
\text { Sakha105 } \\
\quad \text { Hybrid } 1 \\
\text { F. test }\end{array}$ & $\begin{array}{c}68.44 \\
82.22 \\
\text { NS }\end{array}$ & $\begin{array}{c}80.22 \\
82.44 \\
\text { NS }\end{array}$ & $\begin{array}{c}7.81 \mathrm{a} \\
7.14 \mathrm{~b} \\
\star \star\end{array}$ & $\begin{array}{l}7.18 \mathrm{a} \\
6.62 \mathrm{~b} \\
\star \star\end{array}$ & $\begin{array}{l}24.87 b \\
25.88 \mathrm{~b} \\
\star \star \star\end{array}$ & $\begin{array}{c}25.25 \\
25.46 \\
\text { NS }\end{array}$ & $\begin{array}{c}74.20 \mathrm{~b} \\
74.76 \mathrm{a} \\
\star \star\end{array}$ & $\begin{array}{l}73.74 \mathrm{~b} \\
74.30 \mathrm{a}\end{array}$ \\
\hline $\begin{array}{l}I x S \\
I x V \\
S x V \\
I x S x V\end{array}$ & $\underset{\substack{* \star \\
\text { NS } \\
\star}}{*}$ & $\begin{array}{l}\star \star \star \\
\text { NS } \\
\star \star \\
\star \star\end{array}$ & $\begin{array}{l}\star \star \\
\star \star \\
\star \star \\
\star \star\end{array}$ & $\begin{array}{l}\star \\
\star \\
\star \star \\
\star \star\end{array}$ & $\begin{array}{l}\text { NS } \\
\star \star \\
\star \star \\
\star \star\end{array}$ & $\begin{array}{l}N_{\star \star}^{*} \\
\text { NS } \\
\text { NS }\end{array}$ & $\begin{array}{l}\star \star \\
N S \\
\star \star \\
\star \star\end{array}$ & $\begin{array}{l}\text { ** } \\
\text { * } \\
\text { * } \\
\text { * }\end{array}$ \\
\hline
\end{tabular}

${ }^{*},{ }^{* *}$ and NS indicated $\mathrm{P}<0.05 \%, \mathrm{P}<0.01 \%$ and not significant, respectively. Means followed by the same letter are not significantly different at the $5 \%$ level, using Duncan's multiple range test. 
Table (4): Protein content \%, amylose content \%, hulling \%and milling \% of the two rice varieties as affected by irrigation treatment and seedling age in 2013 and 2014 seasons.

\begin{tabular}{|c|c|c|c|c|c|c|c|c|}
\hline \multirow[t]{2}{*}{$\begin{array}{l}\text { Characters } \\
\text { Treatment }\end{array}$} & \multicolumn{2}{|c|}{ Protein content $\%$} & \multicolumn{2}{|c|}{ Amylose content $\%$} & \multicolumn{2}{|c|}{ Hulling \% } & \multicolumn{2}{|c|}{ Milling \% } \\
\hline & 2013 & 2014 & 2013 & 2013 & 2013 & 2014 & 2013 & 2014 \\
\hline $\begin{array}{l}\text { Irrigation (I): } \\
\text { Flooding } \\
\text { Saturation } \\
\text { F. test }\end{array}$ & $\begin{array}{l}7.99 \mathrm{a} \\
7.60 \mathrm{~b} \\
\star \star\end{array}$ & $\underset{* \star}{7.94 \mathrm{a}}$ & $\begin{array}{c}18.67 \\
18.72 \\
\text { NS }\end{array}$ & $\begin{array}{c}18.67 \\
18.66 \\
\text { NS }\end{array}$ & 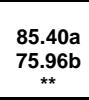 & $\underset{\substack{84.70 a \\
75.73 b}}{* \star}$ & $\begin{array}{c}63.20 \mathrm{a} \\
62.89 \mathrm{~b} \\
\star \star\end{array}$ & $\begin{array}{l}63.27 a \\
62.95 b \\
\star \star\end{array}$ \\
\hline $\begin{array}{l}\text { Seedling age }(\mathrm{S}): \\
15 \text { days } \\
20 \text { days } \\
25 \text { days } \\
\text { F. test }\end{array}$ & $\begin{array}{l}8.42 a \\
8.02 b \\
7.45 c \\
\star \star\end{array}$ & $\begin{array}{l}8.10 \mathrm{a} \\
7.97 \mathrm{~b} \\
6.91 \mathrm{c} \\
\star \star\end{array}$ & $\begin{array}{c}19.37 a \\
18.55 b \\
18.17 c \\
\star \star\end{array}$ & $\begin{array}{l}19.33 \mathrm{a} \\
18.51 \mathrm{~b} \\
18.14 \mathrm{c} \\
\underset{\star \star}{ }\end{array}$ & $\begin{array}{c}85.67 \mathrm{a} \\
84.12 \mathrm{~b} \\
78.25 \mathrm{c} \\
\star \star \star\end{array}$ & $\begin{array}{c}85.03 a \\
83.61 b \\
78.00 c \\
\star \star\end{array}$ & $\begin{array}{c}63.65 a \\
63.34 b \\
62.16 c \\
\star \star \star\end{array}$ & $\begin{array}{c}63.74 a \\
63.40 b \\
62.19 c \\
\star \star \star\end{array}$ \\
\hline $\begin{array}{l}\text { Rice varieties (V): } \\
\text { Sakha105 } \\
\text { Hybrid } 1 \\
\text { F. test }\end{array}$ & $\begin{array}{l}7.65 \mathrm{~b} \\
\underset{\star \star}{* \star}\end{array}$ & $\begin{array}{c}7.60 \mathrm{~b} \\
7.88 \mathrm{a} \\
\star \star\end{array}$ & 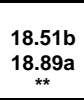 & $\begin{array}{c}18.47 b \\
18.85 a \\
\star \star\end{array}$ & $\begin{array}{l}79.85 \mathrm{~b} \\
85.51 \mathrm{a} \\
\star \star\end{array}$ & $\begin{array}{c}79.18 b \\
85.25 a \\
\star \star\end{array}$ & $\underset{* \star}{62.62 b}$ & $\begin{array}{l}62.71 b \\
63.52 a \\
\star \star\end{array}$ \\
\hline $\begin{array}{l}A \times B \\
A \times C \\
B \times C \\
A \times B \times C\end{array}$ & $\begin{array}{l}\star \star \\
\star \star \\
\star \star \\
\star \star \\
\star \star\end{array}$ & $\begin{array}{l}\star \star \\
\star \star \\
\star \star \\
\star \star\end{array}$ & $\begin{array}{l}\star \star \star \\
\star \star \\
\star \star \\
\star \star\end{array}$ & $\begin{array}{l}\star \star \\
\star \star \\
\star \star \\
\star \star\end{array}$ & $\begin{array}{l}\star \star \\
\star \star \\
\star \star \\
\star \star\end{array}$ & $\begin{array}{l}\star \star * \\
\star \star \\
\star \star \\
\star \star\end{array}$ & $\begin{array}{l}* \star \\
\star * \\
\star * \\
\star \star\end{array}$ & $\begin{array}{l}* \star \\
\star \star \\
\star \star \\
\star \star\end{array}$ \\
\hline
\end{tabular}

${ }^{*},{ }^{* *}$ and NS indicated $\mathrm{P}<0.05 \%, \mathrm{P}<0.01 \%$ and not significant, respectively.

Means followed by the same letter are not significantly different at the $5 \%$ level, using Duncan's multiple range tests.

and number of branches/panicle and grain yield in only the second season, but there were no significant differences between the two varieties on filled grains/panicle and panicle weight in the two seasons. The Egyptian Hybrid1 showed superiority in these characters compared with Sakha 105 inbred rice variety. These results might be attributed to the differences among these cultivars in their genetic constitutions as well as their response to the prevailing environmental conditions. These results are in line with those reported by El-Rewainy et al (2007), Salem (2011), El-Fky (2012) and El-Hefnawy (2012).

Data in Tables (3 and 4) show that there were significant differences between rice varieties on 1000-grain weight in the first season and on hectoliter weight, protein content, amylase content, hulling \% and milling \% in both seasons. The tested Egyptian Hybrid 1 showed superiority in these characters compared with Sakha 105 inbred rice varity. However, conductivity value decreased with Egyptian Hybrid 1. These results were parallel with those reported by El-Maksoud (2008) and El-Hefnawy (2012).

\section{Effect of interaction:}

As shown in Table (5) the interaction among the three factors under study had a significant effect on number of productive tillers $/ \mathrm{m}^{2}$, number of filled grains/panicle and straw yield in both seasons as well as on number of primary branches/panicle in the second season and panicle weight in the first season. Egyptian Hybrid 1 recorded the highest number of productive tillers $/ \mathrm{m}^{2}$ with saturation system under 15 days old seedling (718.33 and 504.00) in both seasons, respectively, 
while Sakha 105 with 15 days under saturation system gave the highest number of branches/panicle (10.68) in the first season. Egyptian Hybrid 1 rice varity recorded the highest number of filled grains/panicle with 20 days old seedling under saturation system (168.88 and 165.47) in the two seasons, respectively. Sakha 105 rice varity with twenty days old seedling under flooding system gave the highest panicle weight $(4.31 \mathrm{~g})$ in the second season.

Table (5): Effect of interaction among irrigation treatment, seedling age and two rice varieties on number of productive tillers $/ \mathrm{m}^{2}$, number of branches/ panicle, number of filled grains/ panicle, panicle weight and straw yield in 2013 and 2014 seasons.

\begin{tabular}{|c|c|c|c|c|}
\hline Characters & $\begin{array}{c}\text { No of } \\
\text { productive } \\
\text { tillers } / \mathrm{m}^{2}\end{array}$ & $\begin{array}{c}\text { No.of branches/ } \\
\text { panicle }\end{array}$ & $\begin{array}{l}\text { No.of filled } \\
\text { grains/panicle }\end{array}$ & Panicle weight \\
\hline I1S1V1 & $608.33 c$ & $8.27 \mathrm{~b}$ & 146.33ab & $3.90 \mathrm{a}$ \\
\hline I1S1V2 & $322.00 f$ & $9.58 \mathrm{ab}$ & 120.67cde & $3.23 a$ \\
\hline I1S2V1 & $526.67 d$ & $8.67 \mathrm{~b}$ & $150.33 a b$ & $3.60 \mathrm{a}$ \\
\hline I1S2V2 & $218.33 h$ & $9.67 a b$ & $114.00 \mathrm{de}$ & $3.91 \mathrm{a}$ \\
\hline I1S3V1 & $541.67 d$ & $9.15 a b$ & $140.00 \mathrm{bc}$ & $3.16 a$ \\
\hline I1S3V2 & $300.00 \mathrm{fg}$ & $8.50 \mathrm{~b}$ & $105.80 \mathrm{e}$ & $3.19 a$ \\
\hline I2S1V1 & $170.00 \mathrm{~h}$ & $10.68 \mathrm{a}$ & $136.73 \mathrm{bcd}$ & $3.90 \mathrm{a}$ \\
\hline I2S1V2 & $718.33 a$ & $8.77 \mathrm{~b}$ & 153.00ab & $3.23 a$ \\
\hline I2S2V1 & $268.33 \mathrm{~g}$ & $9.60 \mathrm{ab}$ & $107.47 \mathrm{e}$ & $3.60 a$ \\
\hline I2S2V2 & $676.67 \mathrm{~b}$ & $9.13 a b$ & $168.88 \mathrm{a}$ & $3.91 \mathrm{a}$ \\
\hline I2S3V1 & $291.67 \mathrm{fg}$ & $7.93 \mathrm{~b}$ & $112.87 \mathrm{de}$ & $3.16 a$ \\
\hline I2S3V2 & $458.33 e$ & $9.07 a b$ & $133.00 \mathrm{bcd}$ & $3.19 a$ \\
\hline F-test & $* *$ & $\star *$ & $\star *$ & NS \\
\hline I1S1V1 & $380.67 d$ & $9.03 a$ & 152.07abc & $2.91 \mathrm{bc}$ \\
\hline I1S1V2 & $415.33 \mathrm{bcd}$ & $9.20 \mathrm{a}$ & 161.13ab & $3.78 \mathrm{ab}$ \\
\hline I1S2V1 & $379.67 d$ & $10.20 \mathrm{a}$ & $94.47 \mathrm{e}$ & $4.31 \mathrm{a}$ \\
\hline I1S2V2 & $518.00 a$ & $9.33 a$ & $134.83 \mathrm{bcd}$ & $3.53 a b$ \\
\hline I1S3V1 & $360.33 d$ & $9.27 a$ & $119.43 \mathrm{de}$ & $3.43 a-c$ \\
\hline I1S3V2 & 397.33cd & $9.40 \mathrm{a}$ & $133.83 \mathrm{~cd}$ & $2.99 \mathrm{bc}$ \\
\hline I2S1V1 & $485.33 a b$ & $8.93 a$ & $103.90 \mathrm{e}$ & $2.91 b c$ \\
\hline I2S1V2 & $504.00 a$ & $10.67 a$ & 145.87a-d & $3.78 \mathrm{ab}$ \\
\hline I2S2V1 & $463.00 \mathrm{abc}$ & $9.07 a$ & 145.47a-d & $4.31 \mathrm{a}$ \\
\hline I2S2V2 & $395.33 \mathrm{~cd}$ & $9.60 \mathrm{a}$ & $165.47 a$ & $3.53 a b$ \\
\hline I2S3V1 & $357.67 \mathrm{~d}$ & $9.20 \mathrm{a}$ & $158.90 a-c$ & $3.43 a-c$ \\
\hline I2S3V2 & $348.67 d$ & $9.73 a$ & $993.83 e$ & $2.99 \mathrm{bc}$ \\
\hline F-test & $\star \star$ & NS & ** & 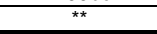 \\
\hline
\end{tabular}

Means followed by the same letter within columns are not significantly different at the $5 \%$ level, using Duncan's multiple range test.

I- $\quad$ irrigation (I1-flooding , I2- saturation) S- seedling age (S1-15 days, S2-20days S3-25 days) V-varieties ( V1-Sakha 105, V2- Hybrid1)

Data in Table (6) show that the interaction among the three factors under study significantly affected germination \%, conductivity, hectoliter weight, protein content, amylase content, hulling $\%$ and milling \% in both seasons as well as 1000 - grain weight was significant in the first season only. Flooding system and 15 days old seedling with Egyptian Hybrid 1 gave the highest germination percentage $(96.67 \%)$ in the first season, while flooding and 20 days old seedling with Egyptian Hybrid 1 varity gave the highest one (96.71\%) in the second season. Flooding irrigation treatments with first age and Egyptian Hybrid 1 produced significantly the highest numerical values of 1000 - 
grain weight $(28.50 \mathrm{~g})$ in the first season, hectoliter weight $(76.51$ and $76.06 \mathrm{~g})$, protein content (8.68 and $8.61 \%)$, amylase content $(20.43$ and $20.44 \%$ ) and milling \% (64.34 and $64.44 \%)$ in both seasons, respectively. While, flooding system with 15 days old seedling and Sakha 105 rice varity gave the highest hulling \% (92.75 and $91.70 \%)$ in 2013 and 2014 seasons, respectively. Electrical conductivity was decreased under flooding with 25 days and Hybrid 1 (6.57) in the first season and under flooding with 20 days and Hybrid 1 (5.69) in the second season.

Table (6): Effect of interaction among irrigation treatment, seedling age and two rice varieties on germination \%, EC, 100-grain weight, hectoliter weight, protein \%, amylase \%, hulling \% and milling \% in 2013 and 2014 seasons.

\begin{tabular}{|c|c|c|c|c|c|c|c|c|}
\hline Characters & $\begin{array}{l}\text { Germinati } \\
\text { on } \%\end{array}$ & $\begin{array}{c}\text { EC } \\
(\mu h o s / g m)\end{array}$ & $\begin{array}{l}1000- \\
\text { Grain } \\
\text { weight } \\
\text { (g) }\end{array}$ & $\begin{array}{l}\text { Hectolit } \\
\text { re } \\
\text { weight }\end{array}$ & $\begin{array}{l}\text { Protein } \\
\text { content }\end{array}$ & $\begin{array}{c}\text { Amylose } \\
\text { content }\end{array}$ & $\underset{\%}{\text { Hulling }}$ & $\begin{array}{l}\text { Milling } \\
\%\end{array}$ \\
\hline & \multicolumn{8}{|c|}{$\frac{1}{2013}$} \\
\hline I1S1V1 & $94.00 a b$ & $8.15 b$ & $23.40 c$ & $75.53 a-c$ & $8.55 a b$ & 18.72de & $92.75 a$ & $63.54 \mathrm{c}$ \\
\hline I1S1V2 & $96.67 a$ & 6.74de & $28.50 \mathrm{a}$ & $76.51 \mathrm{a}$ & $8.68 \mathrm{a}$ & $20.43 a$ & $89.71 c$ & $64.34 a$ \\
\hline I1S2V1 & $90.67 a b$ & 6.99de & $27.23 a b$ & 73.92de & $\mathrm{bc}$ & $17.78 \mathrm{~h}$ & $90.86 \mathrm{~b}$ & $63.35 d$ \\
\hline I1S2V2 & $78.67 b-d$ & $7.79 b c$ & $21.97 c$ & $74.57 b-d$ & $5.44 b$ & $19.16 \mathrm{c}$ & $88.75 \mathrm{e}$ & $64.18 \mathrm{~b}$ \\
\hline I1S3V1 & $54.67 \mathrm{e}$ & 6.83de & $27.83 a b$ & $74.52 b-d$ & $6.94 \mathrm{ef}$ & $17.75 \mathrm{~h}$ & $87.71 \mathrm{~g}$ & $61.06 \mathrm{i}$ \\
\hline I1S3V2 & $78.67 \mathrm{~b}-\mathrm{d}$ & $6.57 \mathrm{e}$ & $21.97 c$ & $75.48 \mathrm{a}-\mathrm{c}$ & $6.95 \mathrm{ef}$ & $18.20 \mathrm{~g}$ & $86.62 \mathrm{~h}$ & $62.73 f$ \\
\hline I2S1V1 & $682.67 \mathrm{bc}$ & $7.41 \mathrm{~cd}$ & $23.40 \mathrm{c}$ & $74.44 \mathrm{~cd}$ & $7.89 d$ & $19.71 \mathrm{~b}$ & $70.77 i$ & $63.06 \mathrm{e}$ \\
\hline I2S1V2 & $64.00 c-e$ & $7.44 \mathrm{~cd}$ & $23.67 c$ & $74.06 \mathrm{de}$ & $8.56 \mathrm{ab}$ & 18.63def & $89.43 d$ & $63.64 \mathrm{c}$ \\
\hline I2S2V1 & $84.00 \mathrm{ab}$ & $7.75 b c$ & $22.37 c$ & 73.79de & $7.16 \mathrm{e}$ & $18.74 d$ & $68.71 \mathrm{k}$ & $62.43 \mathrm{~h}$ \\
\hline I2S2V2 & $82.67 \mathrm{bc}$ & $6.97 \mathrm{de}$ & $27.50 a b$ & $75.65 a b$ & $8.15 c$ & 18.51def & $88.16 f$ & $63.39 d$ \\
\hline I2S3V1 & $62.67 \mathrm{de}$ & $9.69 a$ & $23.17 c$ & 73.00ef & $6.901 \mathrm{f}$ & $18.33 \mathrm{fg}$ & 68.301 & $62.30 \mathrm{i}$ \\
\hline I2S3V2 & $86.67 a b$ & $8.32 b$ & $25.70 \mathrm{~b}$ & $72.30 f$ & $6.95 \mathrm{ef}$ & 18.40 efg & $70.37 j$ & $62.54 \mathrm{~g}$ \\
\hline F-test & * & $\star \star \star$ & ** & ** & $\star \star *$ & ** & $\star \star$ & ** \\
\hline \multicolumn{9}{|c|}{2014} \\
\hline I1S1V1 & $81.38 a b$ & $7.01 b-d$ & $25.77 a$ & $75.07 a-c$ & $8.57 \mathrm{ab}$ & $18.69 \mathrm{~d}$ & $91.70 \mathrm{a}$ & $63.66 \mathrm{c}$ \\
\hline I1S1V2 & $80.00 c$ & $6.20 \mathrm{de}$ & $26.20 \mathrm{a}$ & $76.06 \mathrm{a}$ & $8.61 a$ & $20.44 a$ & $89.07 \mathrm{~b}$ & $64.44 a$ \\
\hline I1S2V1 & $57.33 d$ & $8.37 a$ & $23.93 a$ & $73.46 \mathrm{de}$ & $8.28 d$ & $17.70 \mathrm{~h}$ & $89.28 \mathrm{~b}$ & $63.44 d$ \\
\hline I1S2V2 & $96.71 a$ & $5.69 \mathrm{e}$ & $25.16 \mathrm{a}$ & $74.12 \mathrm{~b}-\mathrm{d}$ & $8.39 c$ & $19.19 \mathrm{c}$ & $88.61 c$ & $64.21 b$ \\
\hline I1S3V1 & $86.00 \mathrm{ab}$ & $6.46 \mathrm{de}$ & $25.69 a$ & $74.06 \mathrm{~b}-\mathrm{d}$ & $6.86 \mathrm{i}$ & $17.77 \mathrm{~h}$ & $87.15 \mathrm{e}$ & $61.14 \mathrm{i}$ \\
\hline I1S3V2 & $78.67 \mathrm{c}$ & $6.81 \mathrm{~cd}$ & $24.93 a$ & $75.02 a-c$ & $6.94 \mathrm{~h}$ & $18.20 \mathrm{~g}$ & $86.41 \mathrm{f}$ & $62.75 f$ \\
\hline I2S1V1 & $80.00 c$ & $6.95 b-d$ & $23.89 a$ & $73.98 \mathrm{~cd}$ & $7.91 \mathrm{f}$ & $19.71 \mathrm{~b}$ & $70.30 \mathrm{~g}$ & $63.15 \mathrm{e}$ \\
\hline I2S1V2 & $76.67 \mathrm{bc}$ & 6.56de & $25.96 a$ & $73.61 \mathrm{de}$ & $8.51 \mathrm{~b}$ & $18.49 \mathrm{e}$ & $89.06 \mathrm{~b}$ & $63.70 \mathrm{c}$ \\
\hline I2S2V1 & $79.67 c$ & 6.56de & $26.97 a$ & 73.33de & $7.09 \mathrm{~g}$ & $18.69 \mathrm{~d}$ & $68.43 h$ & $62.54 \mathrm{~g}$ \\
\hline I2S2V2 & $80.89 c$ & $6.68 d$ & $25.77 a$ & $75.20 \mathrm{ab}$ & $8.12 \mathrm{e}$ & $18.46 \mathrm{e}$ & $88.11 d$ & $63.43 d$ \\
\hline I2S3V1 & $65.33 d$ & 7.70a-c & $25.26 a$ & 72.55ef & $6.89 \mathrm{hi}$ & $18.27 \mathrm{fg}$ & $68.22 \mathrm{~h}$ & $62.31 \mathrm{~h}$ \\
\hline I2S3V2 & $64.00 \mathrm{~d}$ & 7.79ab & $23.76 a$ & $71.84 f$ & $6.95 \mathrm{~h}$ & $18.33 f$ & $70.24 \mathrm{~g}$ & $62.58 \mathrm{~g}$ \\
\hline F-test & ** & ** & NS & ** & ** & ** & ** & ** \\
\hline
\end{tabular}

Means followed by the same letter within columns are not significantly different at the $5 \%$ level, using Duncan's multiple range test.

I- irrigation (I1-flooding, I2- saturation) S- seedling age (S1-15 days, S2-20days S3-25 days) Vvarieties ( V1-Sakha 105, V2- Hybrid1)

\section{CONCLUSION}

From the obtained results of both growing seasons field's study, it was concluded that yield, its components, grain composition, quality and viability of rice crop were increased with utilization of Egyptian Hybrid 1 variety with youngest seedling age (15 day old) and 
flooding treatment under the system of rice intensification (SRI), under the environmental conditions of this research.

\section{REFERENCE}

A. O. A. C. 1990. Official methods of analysis. The Association of Official Analytical Chemists 15th (Edition, Published by Association of Official Analytical Chemists, Arrington, Virginia, USA.)

A. O. S. A., 1986. Association of Official Seed Analysis Seed Vigor Testing Hand Book, No., 32, p. 1.

Aggarwal. N, S. Avtar and Sh. Savita. 2014. Chemical composition, milling and cooking quality of mechanically transplanted rice in relation to seedling age and plant population. Oryza-An International Journal on Rice. Vol.51, Iss:4. P (309-314).

Akhilesh. V, J. K. Singh, A. V. Sen, J. S. Bohra and S. Singh. 2016. Effect of transplanting date and age of seedlings on growth, yield and quality of hybrids under system of rice (Oryza sativa) intensification and their effect on soil fertility. The Indian Journal of Agriculture Sciences. Vol 86, No 5.

Ali, M. S., Hasan., S. Sikder., M. R. Islam and M. H. R. Hafiz. 2013. Effect of Seedling Age and Water management on the performance of Boro rice (Oryza sativa L.) variety BRRI Dhan28. The Agriculturists 11(2): 28-37.

Duncan, D. B. (1955). Multiple range and multiple F test. Biometrics, 11:1-42.

Duraga. K. K. ., P. S. Rao and K. Raju. 2015. Effect of seedling age and spacing schedule on the productivity and quality traits of rice under system of rice intensification (SRI). Journal of Cereal and Oilseeds vol. 6 (3).15-19.

El-Fky. K. A. 2012. Evaluation of some SRI (system of rice intensification) treatments and its effect on rice productivity in North Delta . Ph D. Thesis, Kafrelsheikh Univ., Egypt.

El-Hefnawy., T. M. 2012. The effect of system of rice intensification (SRI) on productivity of rice. M.S. Thesis, Mansoura University, Egypt.

El-Kady, A. A. and A. A. Abdallah. 2004. A study on the effect of planting methods and water management on some grain quality characters of rice. Egyptian J. of Agric. Res. 139-147.

El-Maksoud, M. F. A. 2008. Effect of levels and splitting of N-fertilization on growth, yield components, yield and grain quality of some rice cultivars. Res. J. of Agric. and Bio. Sci. 392-398.

El-Rewainy. I.M . S.A. Hamoud, T.F.Metwaly and S.E. Sedeek. 2007. Response of two rice cultivars to different seedling ages and nitrogen levels. African Crop Science Conference Proceedings Vol. 8. pp. 1937-19410.

Ginigaddara. G. A. S and S.L. Ranamukhaarachchi. 2011. Study of age of seedling at transplanting on growth dynamics and yield of rice under alternating flooding and suspension of irrigation of water 
management. Recent Research in Science and Technology. 3(3): 76-88.

Gilbert, G.A and Spragg, S.P. 1964. lodine Sorption: "Blue Value". In: WHISTLER, R.L. Methods in carbohydrate chemistry: volume IV - starch. London: Academic. p.168-169.

I.S.T.A, 1993. International Rules for Seed Testing. Seed Sci. and Technol., 21: 24-46.

Krishna. A, N. K. Biradarpatil, K. Manjappa and B. B. Channappagoudar. 2008. Evaluation of System of Rice Intensification Cultivation, Seedling Age and Spacing on Seed Yield and Quality in Samba Masuhri (BPT-5204) Rice. Karnataka J. Agric. Sci., 21 (1) (20-25)

Leila R., M. S. Daliri and A. A. Mousavi. 2013. Effect of seedling age on yield and yield component of rice cultivars (Oryza sativa L.). Annals of Biological Research, 4 (2):72-76.

MSTATC, (1990). Microcomputer Program for Design Experiment and Analysis of Agronomic Research Experiments Michigan State Univ.

Naeem.S. M. Maqsood., S. A. Wajid. And M. A. U. Haq. 2011. Impact of nursery seedling density, nitrogen and seedling age on yield and yield attributes of fine rice. Chilean journal of Agricultural Research 71 (3) July- September.

Naidu. G. J., K. T. Rao., A.U. Rao and D. S. Reddy. 2013. Age of seedlings and planting pattern on grain yield, protein content, NPK uptake and post-harvest nutrient status of rice under SRI. Journal of Academia and Industrial Research Vol 2, Is 6.

Pramanik. K and A. K. Bera. 2013. Effect of seedling age and nitrogen fertilizer on growth, chlorophyll content, yield and economics of hybrid rice (Oryza sativa L.). International Journal of Agronomy and Plant Production. Vol., 4 (S), 3489-3499.

Rafaralahy, S. (2002). An NGO perspective on SRI and its origin in Madagascar. In: Assessments of the System of Rice Intensification (SRI): Pro. of the I. Conf., Sanya, China, 17-22.

Rajendran.K, and R. V. Gameasa. 2014. Effect of age of seedling on growth and yield of rice. India Journal of Advances in Plant Research, vol.1 (5):62-66.

Salem. A.K.M., W.M. ElKhoby, A.B. Abou-Khalifa and M. Ceesay. 2011. Effect of nitrogen fertilizer and seedling age on inbred and hybrid rice varieties. American-Eurasian J. Agric. \& Environ. Sci., 11 (5): 640-646.

Shantappa. D, K. Tirupataiah, K. R. Yella, K. Sandhyrami, R. K. Mahenndra and K. Malamasuri. 2014. Yield and water productivity of rice under different cultivation practices and irrigation regimes. Integrated water Resources Management (IWRM). February 19-21. p 938-943.

Singh, K. K.; S. K. Yadav; B. S. Tomar; J. N. Singh and P. K. Singh. 2004. Effect of seedling age on seed yield and seed quality attributes in rice (Oryza sativa L).cv. Pusa Basmati-1. Seed Res. 5-8. 
Singh, R.S. and Singh, S.B. 1998. Response of rice (Oryza sativa) to age of seedlings and levels and time of nitrogen under irrigated condition. Indian J. Agron., 43(4):632-635.

Stoop WA, N. Uphoff and A. Kassam . 2002. A review of agricultural research issues raised by the System of Rice Intensification (SRI) from Madagascar. Opportunities for improving farming systems for resource-poor farmers. Agric. Syst. 71:249-274.

Uphoff, N. 2007. The system of rice intensification: using alternative cultural practices to increase rice production and profitability from existing yield potentials. International Rice Commission Newsletter, Number 55, UN Food and Agriculture Organization, Rome.

Won, Jong-Gun, A. Duok-Jong, K. Se-Jong, C. Chung-Don and L. SangChul. 2008 . Yield and Grain Quality as Affected by Seedling Age in Late Transplanted Rice. Korean Journal of Crop Science. Vol 53, pp.19-23.

$$
\text { الملخص العربي }
$$

تأثير نظام الري و عمر الشتتلات علي انتاجيه و جوده صنفان من الأرز باستخدام نظام

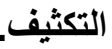

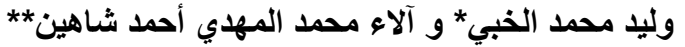

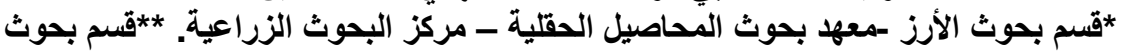

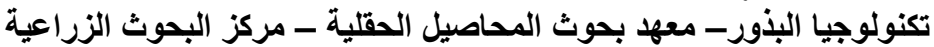

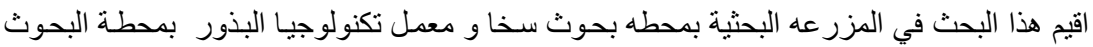

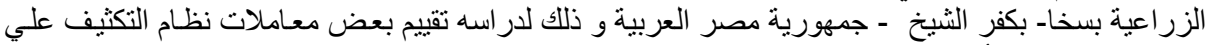

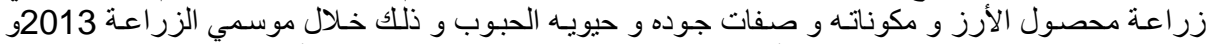

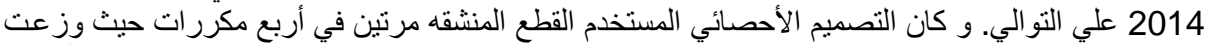

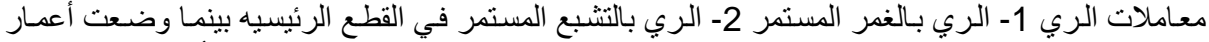

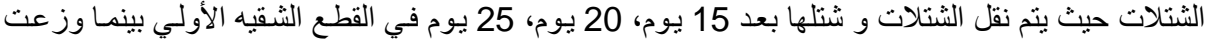
الأصناف و هي سخا 105 ، هجين 1 في القطع الثقين الثنانيه.

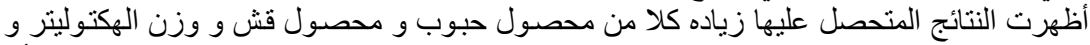

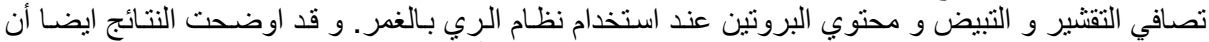

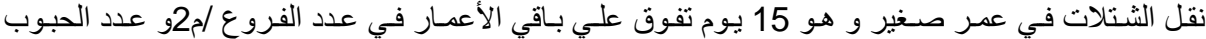

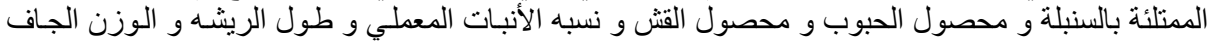

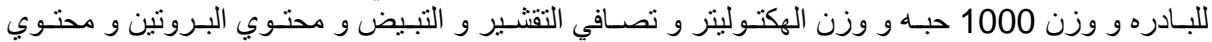

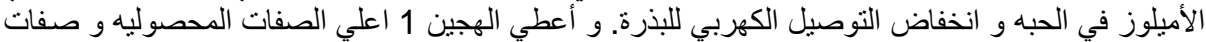

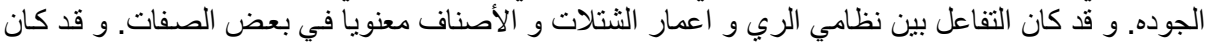

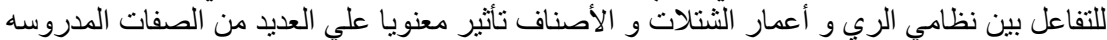

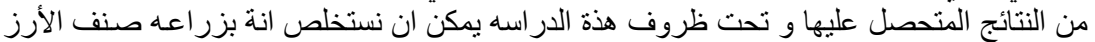

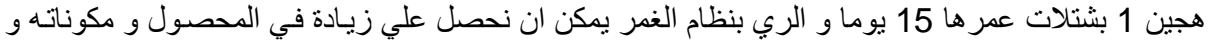
تحسين صفات الجودة و الحيوية للحبوب. 